\title{
DISCRETE FRACTIONAL FOURIER TRANSFORM
}

\author{
Soo-Chang Pei ${ }^{1} \quad$ Min-Hung Yeh ${ }^{2}$ \\ ${ }^{1}$ Department of Electrical Engineering, National Taiwan University, Taipei, Taiwan, R. O. C. \\ Email address: pei@cc.ee.ntu.edu.tw \\ ${ }^{2}$ Department of Electrical Engineering, National Taiwan University, Taipei, Taiwan, R. O. C. \\ Email address: yeh@csp.ee.ntu.edu.tw
}

\begin{abstract}
The continuous fractional Fourier transform (FRFT) represents a rotation of signal in time-frequency plane, and it becomes an important tool for signal analysis. A discrete version of fractional Fourier transform has been developed but its results do not match those of continuous case. In this paper, we propose a new version of discrete fractional Fourier transform (DFRFT). This new DFRFT will provide similar transforms as those of continuous fractional Fourier transform and also hold the rotation properties.
\end{abstract}

\section{INTRODUCTION}

Successive application of the Fourier transform $\mathcal{F}$ on the signal $x(t)$ yields $\mathcal{F}^{2}[x(t)]=x(-t), \mathcal{F}^{3}[x(t)]=$ $X(-w), \mathcal{F}^{4}[x(t)]=x(t)$. Based upon this notation, the Fourier transform can be interpreted as a $\frac{\pi}{2}$ rotation of signal in the time-frequency plane. A generalization of Fourier trasform, FRFT, is treated as a rotation of signal in the time-frequency plane, so it satisfies the following rotation properties:

1. Zero rotation: $\Re_{0}=I$

2. Additivity of rotation: $\Re_{\alpha} \Re_{\beta}=\Re_{\alpha+\beta}$

3. Consistency with Fourier transform: $\Re_{\frac{\pi}{2}}=\mathcal{F}$

4. $2 \pi$ rotation: $\Re_{2 \pi}=I$

where $\Re$ indicates the rotation operation in the timefrequency plane. Parameters $\alpha$ and $\beta$ are the rotation angles. The transform kernel of continuous fractional Fourier transform (FRFT) is defined as[4]:

$$
K_{\alpha}(t, u)=\sqrt{\frac{1-j \cot \alpha}{2 \pi}} e^{j \frac{t^{2}+u^{2}}{2} \cot \alpha-j u t \csc \alpha}
$$

Using the kernel of FRFT, the FRFT of the signal $x(t)$, with angular parameter $\alpha$, is computed as:

$$
X_{\alpha}(u)=\int_{-\infty}^{\infty} x(t) K_{\alpha}(t, u) d t
$$

The signal $x(t)$ can be recovered form a FRFT operation with angular parameter $(-\alpha)$;

$$
x(t)=\int_{-\infty}^{\infty} X_{\alpha}(u) K_{-\alpha}(u, t) d u
$$

The main properties of FRFT is well discussed in [4]. Figure 1 shows the FRFT of the rectangle window function $(x(t)=$
1 for $|t| \leq 2$; otherwise $x(t)=0$ ) for various angles. The real parts of FRFT or DFRFT in this paper are plotted by solid lines, and the imaginary parts of FRFT or DFRFT are plotted by dashed lines.

\section{THE ROATAIONAL CONCEPT IN DISCRETE CASE}

The discrete fractional Fourier transform (DFRFT) must obey the rotation rules as those of FRFT. These rotational properties can be realized by the power laws of kernel matrix in discrete case. The power of DFRFT kernel and the rotation angles in DFRFT essentially mean the same thing. In order to avoid ambiguity, the Greek letters (for example: $\alpha, \beta$ ) are used to denote the rotation angles in the time-frequency planes and the English letters are used to denote the power values of the DFT kernel matrices in this paper. The relationships between the fractional power of DFT kernel matrix and the rotation angle of the DFT operation are listed below:

$$
\begin{aligned}
\Re_{\alpha} & =\mathbf{F}^{\frac{2 \alpha}{\pi}} \\
\mathbf{F}^{t} & =\Re_{\frac{t \pi}{2}}
\end{aligned}
$$

The method to compute DFRFT proposed in [2] and [5] is direct computing the fractional power of Fourier transform kernel. The fractional power of Fourier transform kernel can be calculated by equation (6):

$$
\mathbf{F}^{t}=\alpha_{0}(t) \mathbf{F}^{0}+\alpha_{1}(t) \mathbf{F}^{1}+\alpha_{2}(t) \mathbf{F}^{2}+\alpha_{3}(t) \mathcal{F}^{3}
$$

where

$$
\alpha_{i}(t)=\frac{1}{4} \sum_{k=1}^{4} e^{j \frac{\pi}{2}(t-i) k}
$$

Applying the above defined kernel to signal, the DFRFT of the signal $x(t)$ is compute as:

$\mathbf{F}^{t}[x(n)]=\alpha_{0}(t) x(n)+\alpha_{2}(t) x(-n)+\alpha_{1}(t) \mathcal{X}(n)+\alpha_{3}(t) \mathcal{X}(-n)$

where $\mathcal{X}(n)$ is the DFT of signal $x(n)$. Equation (8) indicates the DFRFT of the signal $x(t)$ are the angular combination of the four parts: the original signal $x(n)$, its DFT, a circular flipped version of the signal $x(n)$ and a circular flipped version of its DFT. Although this definition of DFRFT obey the rotation principle as FRFT and can be computed through the DFT fast algorithm. But this DFRFT does not provide the same transforms as those of FRFT. The relationship between DFRFT and FRFT is unknown. 


\section{NEW DISCRETE FRACTIONAL FOURIER TRANSFORM}

The development of our DFRFT is based upon the eigendecomposition of the DFT kernel matrix. To begin with, we will review the properties of the eigenvalues and eigenvectors of the DFT kernel matrix. It has been shown in [6] that the kernel matrix of DFT has only four distinct eigenvalues: $[1,-j,-1, j]$. The multiplicities of the eigenvalues of the DFT have also been derived in [6] and are shown again in Table 1. Because the eigenvectors of DFT kernel $\mathbf{F}$

\begin{tabular}{|c|c|c|c|c|}
\hline$N$ & 1 & $-j$ & -1 & $j$ \\
\hline $4 m$ & $m+1$ & $m$ & $m$ & $m-1$ \\
\hline $4 m+1$ & $m+1$ & $m$ & $m$ & $m$ \\
\hline $4 m+2$ & $m+1$ & $m$ & $m+1$ & $m$ \\
\hline $4 m+3$ & $m+1$ & $m+1$ & $m+1$ & $m$ \\
\hline
\end{tabular}

Table 1. Multiplicities of the eigenvalues of DFT kernel matrix

are not uniquely determined, the eigenvectors corresponding to the same eigenvalues construct a vector space. Every vector spanned by the eigenvectors corresponding to the same eigenvalues is also an eigenvector of $\mathbf{F}$. In [2], B. W. Dickinson and K. Steiglits introduced a commuting matrix $\mathbf{S}$ to compute the eigenvectors of $\mathbf{F}$ with real values. The definition of matrix $\mathbf{S}$ is listed as follows:

$$
\mathbf{S}=\left[\begin{array}{cccccc}
2 & 1 & 0 & 0 & \cdots & 1 \\
1 & 2 \cos \omega & 1 & 0 & \cdots & 0 \\
0 & 1 & 2 \cos 2 \omega & 1 & \cdots & 0 \\
\vdots & \vdots & \vdots & \ddots & & \vdots \\
1 & 0 & 0 & 0 & \cdots & 2 \cos (N-1) \omega
\end{array}\right]
$$

where $\omega=\frac{2 \pi}{N}$. Matrix $\mathbf{S}$ commutes with matrix $\mathbf{F}$ and it satisfies the following commutative property:

$$
\mathrm{SF}=\mathrm{FS}
$$

The eigenvectors of $\mathbf{S}$ are also the eigenvectors of $\mathbf{F}$, but they correspond to different eigenvalues. Because matrix $\mathbf{S}$ is a symmetric matrix, the eigenvalues of matrix $\mathbf{S}$ are all real and the eigenvectors are orthonormal each other. The eigen-decomposition of matrix $\mathbf{S}$ is written as:

$$
\mathbf{S}=\sum_{k=0}^{N-1} \gamma_{k} \mathbf{v}_{k} \mathbf{v}_{k}^{*}
$$

where $\gamma_{k}$ is the eigenvalue of the matrix $\mathbf{S}$, and $\mathbf{v}_{k}$ is the eigenvector of the matrix $\mathbf{S}$ corresponding to the eigenvalue $\gamma_{k}$. The eigen-decomposition of DFT kernel matrix $\mathbf{F}$ is written as:

$\mathbf{F}=\sum_{k \in E_{1}} \mathbf{v}_{k} \mathbf{v}_{k}^{*}+\sum_{k \in E_{2}}(-j) \mathbf{v}_{k} \mathbf{v}_{k}^{*}+\sum_{k \in E_{3}}(-1) \mathbf{v}_{k} \mathbf{v}_{k}^{*}+\sum_{k \in E_{4}}(j) \mathbf{v}_{k} \mathbf{v}_{k}^{*}$

where $E_{1}$ is the set of indices for eigenvectors belongs to $\lambda_{k}=1 . \quad E_{2}$ for $\lambda_{k}=-j$ and so on. The eigenvector obtained from matrix $\mathbf{S}$ can be regarded as the discrete Hermite function for the following reasons:

1. The eigenvectors obtained from matrix $\mathbf{S}$ are also the eigenvectors of discrete Fourier transform kernel.

2. The eigenvectors obtained from matrix $\mathbf{S}$ are all real function.
3. The eigenvectors of matrix $\mathbf{S}$ construct an orthonormal basis.

Based upon the extensive numerical evidence, it can be found that the numbers of sign-changes in the DFT-shifted eigenvectors of matrix $\mathbf{S}$ are $0,1,2, \cdots, N-1$.

Eigenvalues Assignment Rule

The power of matrix can be calculated from its eigendecomposition and directly make the powers for the eigenvalues. But the ambiguity presented in the fraction powers of eigenvalues. The eigenvalues of FRFT are $e^{-j n \alpha}[3]$, so the ambiguity existed in the fractional power of the eigenvalues is solved by the order of Hermite function. Because the eigenvectors of matrix $S$ is regarded as the discrete version of Hermite function, the eigenvalues of $\mathbf{F}$ can be determined based upon the corresponding order of discrete Hermite function. The $n$-th order Hermite function have $n$ zeros, the number of sign-changes in the eigenvectors of matrix $\mathbf{S}$ can be used determined to the Hermite order which the eigenvectors of $\mathbf{F}$ correspond to. If the eigenvector has $k$ sign-changes, it is regarded as the $k$-th order discrete Hermite function and it corresponds to the eigenvalue $e^{-j k \alpha}$. But the numbers of sign-changes in an $N$-point eigenvector can have at most $(N-1)$. If the eigenvalue of this eigenvector is assigned to $e^{-j(N-1) \alpha}$, the multiplicities of eigenvalues of DFT will not match those shown in Table 1 for the two cases: $N=4 m$ and $N=4 m+2$. This eigenvalue of this eigenvector should be assigned to the value $e^{-j \alpha N}$. Table 2 shows the eigenvalues assignment rule for various cases of $N$.

\begin{tabular}{|c|l|}
\hline $\mathbf{N}$ & the eigenvalues \\
\hline $4 m$ & $e^{-j \frac{k \pi}{2}}, k=0,1,2, \cdots, 4 m-2,4 m$ \\
\hline $4 m+1$ & $e^{-j \frac{k \pi}{2}}, k=0,1,2, \cdots, 4 m-1,4 m$ \\
\hline $4 m+2$ & $e^{-j \frac{k \pi}{2}}, k=0,1,2, \cdots, 4 m, 4 m+2$ \\
\hline $4 m+3$ & $e^{-j \frac{k \pi}{2}}, k=0,1,2, \cdots, 4 m+1,4 m+2$ \\
\hline
\end{tabular}

Table 2. The eigenvalues assignment rule of DFT kernel matrix

After the eigenvalues of DFT kernel is determined, the transform kernel of DFRFT can be easily defined by taking fractional powers of the eigenvalues.

$$
\begin{aligned}
\Re_{\alpha} & =\mathbf{F}^{\frac{2 \alpha}{\pi}} \\
& = \begin{cases}\sum_{k=0}^{N-1} e^{-j k \alpha} \mathbf{v}_{k} \mathbf{v}_{k}^{*} & \mathrm{~N} \text { is odd } \\
\sum_{k=0}^{N-2} e^{-j k \alpha} \mathbf{v}_{k} \mathbf{v}_{k}^{*}+e^{-j N \alpha} \mathbf{v}_{N-1} \mathbf{v}_{N-1}^{*} & \mathrm{~N} \text { is even }\end{cases}
\end{aligned}
$$

where $\mathbf{v}_{k}$ is the eigenvector obtained from matrix $\mathbf{S}$, and it has $k$ sign-changes in the DFT-shifted case.

The DFRFT of signal $x(n)$ can be computed through equation (12):

$$
X_{\alpha}(n)=\Re_{\alpha} x(n)=\mathbf{F}^{\frac{2 \alpha}{\pi}} x(n)=\mathbf{V D}^{\frac{2 \alpha}{\pi}} \mathbf{V}^{*} x(n)
$$

The signal $x(n)$ can also be recovered from its DFRFT through a operation with parameter $(-\alpha)$ as the continuous case.

$$
x(n)=\Re_{-\alpha} X_{\alpha}(n)=\mathbf{V D}^{-\frac{2 \alpha}{\pi}} \mathbf{V}^{*} X_{\alpha}(n)
$$


Example: Here, we deal with the rectangle window with period $[-2,2]$. The sampling interval is $T_{s}=4 / 13$, and the number of point is $N=73$, The discrete data become: ( $N=$ $73, f(k)=1,-6 \leq k \leq 6$; otherwise $f(k)=0)$. Figure 2 shows the results of DFRFT proposed in [5]. Figure 3 shows the results of new DFRFT. Comparing the results shown from Figure 1 to Figure 3, we can find the transform results in Figure 3 are very similar to those shown in Figure 1. But the curves of Figure 3 are much smoother than those of Figure 1, it is for the reason that only 73 points of data is used in our experiments. The resolution of DFRFT is not sufficient, so it cannot reveal the value-changes in the transform results as those of continuous case.

The development of this DFRFT is based upon the eigendecomposition of DFT kernel matrix $\mathbf{F}$. The main difference of our method from that developed in [5] is with different eigenvalues assignment rule. The method proposed in [5] assigns the fractional powers of eigenvalues of $\mathbf{F}$ only to four values: $e^{-j \alpha}, e^{-j 2 \alpha}, e^{-j 3 \alpha}, e^{-j 4 \alpha}$, and it ignores the implicit orders existed in the eigenvectors (regarded as discrete Hermite function) of matrix $\mathbf{F}$.

\section{PROPERTIES OF DFRFT}

Most of the properties of this DFRFT are inherited from DFT and FRFT. Properties of the new DFRFT are discussed below:

1. Unitary: The DFRFT operator $\Re_{\alpha}$ inherited the unitary of DFT kernel.

$$
\Re_{\alpha}^{*}=\Re_{\alpha}^{-1}=\Re_{-\alpha}
$$

In other word, this transform preserve the signal energy.

$$
\left\|\Re_{\alpha}[x(n)]\right\|=\|x(n)\|
$$

2. Angle additivity: The rotation with angular parameter $\alpha$ followed by another rotation with angular parameter $\beta$ is equivalent to the rotation with parameter $\alpha+\beta$.

$$
\Re_{\alpha} \Re_{\beta}=\Re_{\alpha+\beta}
$$

3. Time Inversion:

$$
\Re_{\alpha} x(-n)=X_{\alpha}(-n)
$$

4. Periodicity: The transform kernel and the result of transform are periodic with period $2 \pi$.

$$
\Re_{\alpha+2 \pi}=\Re_{\alpha}
$$

5. Symmetric: The transform kernel is a symmetric matrix.

$$
\Re_{\alpha}(a, b)=\Re_{\alpha}(b, a)
$$

6. Eigenfunction: This transform similar to the case of FRFT has a Hermite-like function as its eigenvector.

7. Parity: If signal $x(n)$ is even, the transform $X_{\alpha}(n)$ is also even. If signal $x(n)$ is odd, the tranisform $X_{\alpha}(n)$ is also odd.

\section{CONCLUSIONS}

The DFRFT proposed in this paper can provide the similar transforms as those of continuous case, and it also holds the rotation properties. This DFRFT provide a method for implementing DFRFT in digital electronic system.

\section{REFERENCES}

[1] G. Sansone, Orthogonal Functions Interscience Publisher Inc., 1959.

[2] B. W. Dickinson and K. Steiglitz, "Eigenvectors and functions of the discrete Fourier transform," IEEE Trans. Acoust., Speech, and Signal Process., vol. ASSP-30, pp. 25-31, February 1982.

[3] H. M. Ozaktas and B. Barshan, "Convolution, filtering, and multiplexing in fractional Fourier domains and their relationship to chirp and wavelet transforms," $J$. Opt. Soc. Amer. A, vol. 11, February 1994.

[4] L. B. Almeida, "The fractional Fourier transform and time-frequency representation," IEEE Trans. Signal Process., vol. 42, pp. 3084-3091, November 1994.

[5] Balasubramaniam Santhanam and J. H. McClellan, "The DRFT - A rotation in time-frequency space," Proceedings of ICASSP 1995, pp. 921-924.

[6] J. H. McClellan and T. W. Parks, "Eigenvalue and eigenvector decomposition of the discrete Fourier transform," IEEE Transactions on Audio and Electroacoustics., AU-20, pp. 66-74, March 1972.
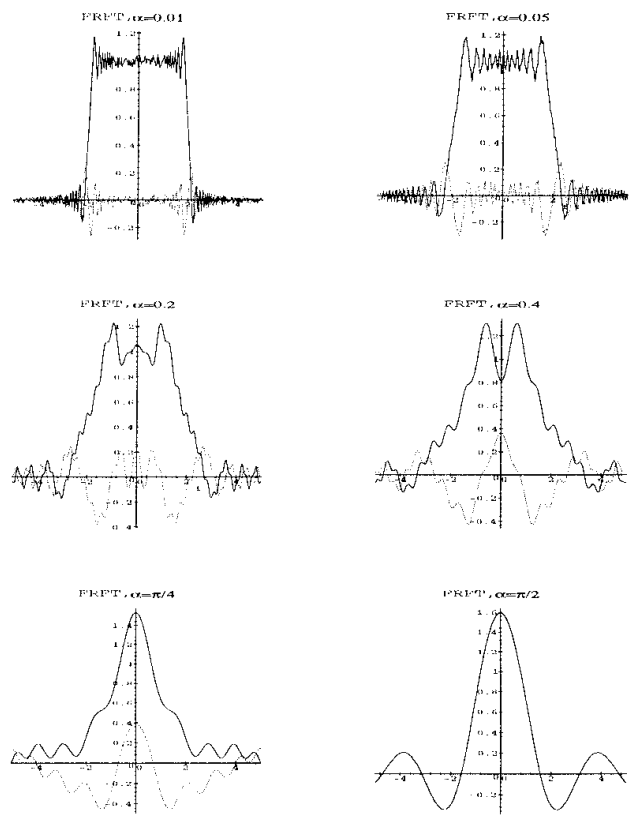

Figure 1. The FRFT of rectangle window 

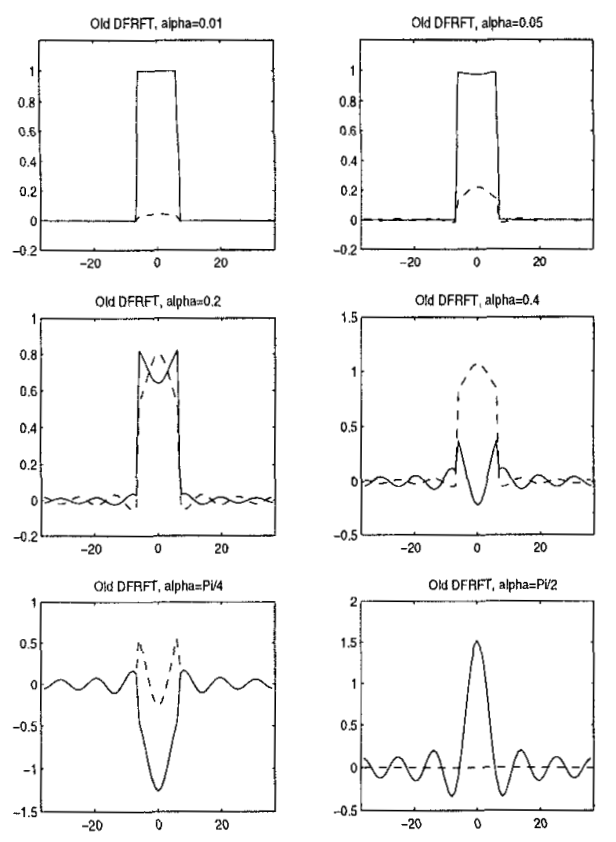

Figure 2. The old DFRFT of the rectangle window function for various angles
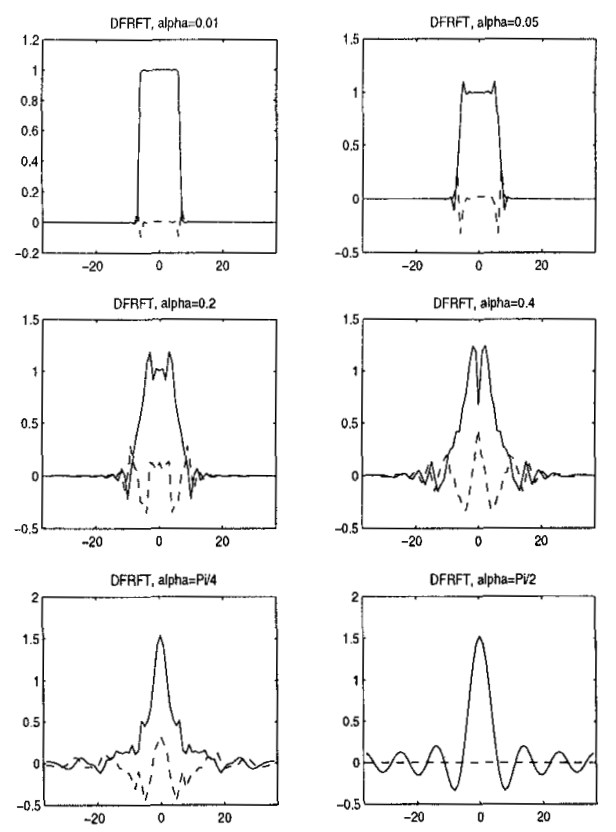

Figure 3. The new DFRFT of the rectangle window function for various angles 